\title{
Subcellular localization of several structurally different tyrosine kinase inhibitors
}

\author{
Richard J. Honeywell*, Sarina M. Hitzerd, G.A.M. Kathmann, Godefridus J. Peters \\ Dept of Medical Oncology, VU University Medical Center, PO Box 7057, 1007 MB Amsterdam, The Netherlands \\ *Corresponding Author: E-mail: r.honeywell@vumc.nl.
}

Received: March 06, 2018; Revised: May 21, 2018; Available online: July 16, 2018

\begin{abstract}
Protein tyrosine kinases form an important target for a new class of anticancer drugs, the tyrosine kinase inhibitors (TKIS). Recently we demonstrated that sunitinib, an inhibitor of the membrane-associated vascular endothelial growth factor receptor (VEGFR), is trapped in lysosomes which isolates the drug from its intended target. Therefore we investigated whether this also holds for other TKIs, targeted against different protein kinases. For this purpose we used the ProteoExtractR kit, which enables a subcellular extraction separating cellular proteins into four distinct fractions covering the cytosol, membranes and membrane organelles (including lysosomes), nuclear proteins and the cytoskeleton. Since TKIs are 98-100\% protein bound we used this property to study their subcellular distribution and used Caco-2 cells as a model. As expected after 2 hours exposure sunitinib was trapped in cytosol (58\%) and organelles (42\% including lysosomes). Crizotinib, an inhibitor of ALK-EML4, showed a similar distribution. However, erlotinib, an inhibitor of the epidermal growth factor receptor (EGFR) showed a very low cellular accumulation and was limited to the organelle fraction. In contrast, the other EGFR inhibitor, gefitinib was predominantly located in the cytosolic (39\%) and membrane fraction (44\%). Sorafenib, another VEGFR inhibitor was predominantly located in the organelle fraction (85\%) and cytosol (15\%) after 2 hours, while after 24 hours distribution decreased (9.9 fold) with a slight shift. Dasatinib, an inhibitor of BCR-Abl was located only in the cytosol (100\%). In general localization after 24 hours was comparable, albeit several small changes were seen. In conclusion protein fractionation with the ProteoExtractR Subcellular Proteome Extraction kit demonstrated large differences in TKI levels in various cellular organelles, with a pattern in agreement with lysosomal accumulation of sunitinib.
\end{abstract}

\section{Keywords}

tyrosine kinase inhibitors; lysosomal accumulation; subcellular distribution; sunitinib; erlotinib; dasatinib

\section{Introduction}

Protein tyrosine kinases (PTKs) regulate important signaling processes specific for the cancer cell [1]. Because of this important role a new class of drugs, the tyrosine kinase inhibitors (TKIs) have been developed [2,3]. The targets for these drugs are receptors located either on or in the membrane or intracellularly. This holds both for tumor cells and tumor-associated endothelial cells [4]. A decreased uptake or increased efflux may limit the cellular drug accumulation, and the efficacy of these drugs [5]. Moreover, inhibition may be reversible or irreversible; especially novel third or fourth generation TKIs display irreversible enzyme inhibition [6]. Recently we demonstrated that sunitinib, an inhibitor of the 
Vascular endothelial growth factor receptor (VEGFR) accumulates in lysosomes [7], while preliminary experiments demonstrated a similar distribution for crizotinib, an ALK-EML4 and CMET inhibitor [5]. In contrast, erlotinib an inhibitor of the epidermal growth factor receptor (EGFR) showed a very low cellular uptake limited to the membrane [8]. Therefore, we wondered whether drug accumulation or drug distribution would be related to their efficacy. Since TKIs are $98-100 \%$ plasma protein bound $[9,10]$ we also wondered whether this is reflected cellularly and whether this would affect the distribution and accumulation of a TKI. For this purpose, we used methodology, which is commonly applied in the field of proteomics, in which the isolation and analysis of proteins based on their cellular location is a rapidly expanding field. However, the different proteins that make up a cellular environment differ greatly and are difficult to isolate due to size, hydrophobicity and basicity. Using the differential solubility of different proteins in the various subcellular compartments has led to the separation of four distinct fractions, the cytosolic fraction, the membrane and organelle fraction, cellular nucleus, and the cytoskeleton, Since we used this approach to successfully characterize the cellular distribution of a lipophilic prodrug of cytarabine [11] we applied the same methodology to determine the cellular distribution of TKIs. Since TKIs are almost completely bound to proteins, a cellular fractionation of cells incubated with drug for a suitable time period should also provide fractionation of the accumulated TKI. Simple extraction of each fraction should then provide data on the distribution of the TKI within the cell. Using a previously developed analytical liquid chromatography - mass spectrometry method [12] the exact proportion of TKI can be determined in each fraction with a high degree of specificity. Using this technique, we determined the subcellular distribution of six TKIs (sunitinib, crizotinib, erlotinib, gefitinib, sorafenib and dasatinib) within the colon cancer cell line, Caco-2. These six inhibitors were chosen based on their FDA status and the extent to which they are used in the clinic. Each of the selected molecules is the first line treatment in their specific area and regularly used for clinical patients.

\section{Materials and methods}

\section{Cell culture}

The Caco-2 cell line, originating from a colorectal adenocarcinoma, was cultured at $37{ }^{\circ} \mathrm{C}, 5 \% \mathrm{CO}_{2}$ and $100 \%$ humidity, in Dulbecco's modified Eagle medium (DMEM) supplemented with $10 \%$ fetal bovine serum (FBS) and $20 \mathrm{mM}$ Hepes [13]. For the fractionation Caco-2 cells were seeded in $6 \mathrm{ml}$ DMEM culture medium with $1 \%$ penicillin/streptomycin in $25 \mathrm{~cm}^{2}$ flasks. Cells were allowed to attach and grow for 72 hours (aiming at an ultimate cell number of $3-5 \times 10^{6}$ cells). Attached cells were than exposed to either $10 \mu \mathrm{M}$ erlotinib, gefitinib, sorafenib, sunitinib, crizotinib or $1 \mu \mathrm{M}$ dasatinib for 2 and 24 hours.

Next, medium was aspirated and cells were washed three times with $10 \mathrm{ml}$ ice cold PBS. To each flask $500 \mu \mathrm{l}$ of trypsin/EDTA was added and cells were incubated for about $5-10$ minutes at $37{ }^{\circ} \mathrm{C}, 5 \% \mathrm{CO}_{2}$ and $100 \%$ humidity. When cells were detached, the cells were resuspended with $1.5 \mathrm{ml}$ ice cold phosphate buffered saline and transferred into eppendorf tubes. After centrifugation, the supernatant was discarded and the pellets resuspended in $200 \mu$ l of water for fractionation.

\section{Cellular fractionation}

The ProteoExtract ${ }^{\circledR}$, subcellular proteome extraction kit from Calbiochem (Cat. No. 539791), was used to fractionate the cell pellets. The differential solubility of proteins in the various subcellular fractions was used to isolate these fractions by the addition of four sequential extraction buffers. Each buffer specifically targets a subcellular compartment while maintaining structural integrity of the cell [13]. When correctly applied these fractions are distinct and can separate the different subcellular compartments with minimal 
to no mixing. Cell suspensions were centrifuged at $100-300 \mathrm{~g}$ for 10 minutes at $4{ }^{\circ} \mathrm{C}$, then the supernatant was removed. Next, the cell pellets were washed with $2 \mathrm{ml}$ ice cold washing buffer, gently resuspended, incubated for 5 minutes at $4{ }^{\circ} \mathrm{C}$ and centrifuged at $100-300 \mathrm{~g}$ at $4{ }^{\circ} \mathrm{C}$ for 10 minutes. The supernatant was discarded and the washing step was repeated once more. The resulting cell pellets were used to separate 4 distinct cellular compartments. The initial stage of the extraction releases the cytosolic proteins from the cell, by addition of $1 \mathrm{ml}$ of ice cold extraction buffer I with $5 \mu \mathrm{l}$ protease inhibitor cocktail (PIC) to each cell pellet, which were incubated for 10 minutes at $4{ }^{\circ} \mathrm{C}$ and centrifuged at $500-1000 \mathrm{~g}$ at $4{ }^{\circ} \mathrm{C}$ for 10 minutes. The supernatant was then transferred quantitatively into a clean labelled tube without disturbing the pellet (fraction 1, representing the cytosolic proteins) and stored at $-80^{\circ} \mathrm{C}$ for future analysis. Subsequently, $1 \mathrm{ml}$ of ice cold extraction buffer II with $5 \mu \mathrm{I}$ PIC was added to each pellet to solubilize the membrane and organelles fraction, incubated for 30 minutes at $4{ }^{\circ} \mathrm{C}$ and then centrifuged at $5000-6000 \mathrm{~g}$ at $4{ }^{\circ} \mathrm{C}$ for 10 minutes. The supernatant was transferred quantitatively into a clean tube without disturbing the pellet (fraction 2 representing the membrane and organelle proteins) and stored at $-80^{\circ} \mathrm{C}$ for future analysis. Next, $500 \mu \mathrm{l}$ of ice cold extraction buffer III with $5 \mu \mathrm{I}$ PIC and $1.5 \mu \mathrm{l}$ benzonase nuclease was added to each pellet, incubated for 10 minutes at $4{ }^{\circ} \mathrm{C}$ and centrifuged at $6800 \mathrm{~g}$ at $4{ }^{\circ} \mathrm{C}$ for 10 minutes. The supernatant was transferred into a clean tube without disturbing the pellet, containing nuclear proteins (fraction 3 ) and stored at $-80^{\circ} \mathrm{C}$ for future analysis. The last stage was to add $500 \mu \mathrm{l}$ of extraction buffer IV with $5 \mu \mathrm{IIC}$ to each cell pellet, obtaining the components of the cytoskeleton (fraction 4) and stored at $-80^{\circ} \mathrm{C}$ for future analysis.

\section{Data analysis by LC-MS/MS}

All samples of the subcellular fractionation were measured using liquid chromatography coupled to mass spectrometry to determine the amount of drug (TKI) present using a previously developed method for biological materials. Briefly, $20 \mu \mathrm{l}$ of each supernatant fraction was transferred quantitatively into a round bottomed 96 -well plate and $80 \mu \mathrm{l}$ of ice cold acetonitrile added. Each plate was covered with a plastic foil and allowed to stand for 10 minutes. Subsequently the 96 -well plate was centrifuged at $1500 \mathrm{~g}$ at $4{ }^{\circ} \mathrm{C}$ for 10 minutes. Next, $50 \mu$ l of each sample was transferred to a clean conical 96-well plate and covered with a NUNC well plate seal. Lastly, $1 \mu \mathrm{l}$ of each sample was injected into the LC-MS/MS under the following conditions. A mobile phase containing $0.578 \mathrm{~g}$ ammonium acetate, $375 \mathrm{ml}$ MilliQ water, $125 \mathrm{ml}$ methanol, $0.1 \%$ isopropanol and $1000 \mathrm{ml}$ acetonitrile with a $\mathrm{pH}$ of 7.8 was used at $350 \mu \mathrm{l} / \mathrm{min}$. Before use, the mobile phase was filtered using a nylon filter paper of $0.2 \mu \mathrm{m}$ and subsequently degassed for 10 minutes. Chromatography was performed on a Phenomenex prodigy ODS 3 column (100x2 mm, $3 \mu \mathrm{m})$.

\section{Data analysis}

Concentration of compounds determined by LC-MS/MS in each of the fractions was normalized to total volume of the collected fraction and quantified as accumulated drug within each fraction (fmol). Protein content of each fraction was determined and data normalised to $\mathrm{fmol} / \mathrm{mg}$ protein. The percentage of drug observed in each fraction was then determined in relation to the total drug accumulation (the sum total of all fractions per compound). Fold changes between the 2 hour and 24 hour accumulation time points were determined as the ratio of total drug accumulated at the 2 hour mark to the total accumulated at the 24 hour time mark. 


\section{Results}

Using the proteome extraction kit we managed to separate the proteins bound into representative fractions of the cellular compartments, even when accumulated at very low levels. Sunitinib has previously been shown to accumulate within the lysosomal compartment of the cellular makeup, where it can reach very high concentrations. Hence, sunitinib was used as a control for the occurrence of lysosomal accumulation. As expected after 2 hours sunitinib was predominantly located in the cytosol (57.5\%), membranes and membrane organelles (42.1\%) (e.g. lysosomes). After 24 hours no distribution change was observed, although the absolute amount of sunitinib decreased 3.9 fold (Tables 1 and 2; Figure 1).

Table 1. Subcellular distribution of 6 tyrosine kinase inhibitors

\begin{tabular}{|c|c|c|c|c|c|c|c|}
\hline & $\begin{array}{c}\text { Time } \\
\text { (hours) }\end{array}$ & Sunitinib & Crizotinib & Erlotinib & Gefitinib & Sorafenib & Dasatinib \\
\hline \multirow{2}{*}{ Cytosolic Fraction (1) } & 2 & 57.5 & 58.3 & 10.1 & 38.8 & 15.3 & 100 \\
\hline & 24 & 56.6 & 41.7 & 0.0 & 56.3 & 0.0 & 55.6 \\
\hline \multirow{2}{*}{ Membrane Fraction (2) } & 2 & 42.1 & 41.7 & 7.1 & 44.4 & 84.7 & 0.0 \\
\hline & 24 & 40.2 & 56.4 & 0.0 & 30.0 & 60.4 & 27.9 \\
\hline \multirow{2}{*}{ Organelles Fraction (3) } & 2 & 0.4 & 0.0 & 3.6 & 16.8 & 0.0 & 0.0 \\
\hline & 24 & 2.7 & 2.0 & 0.0 & 11.0 & 13.9 & 0.0 \\
\hline \multirow{2}{*}{ Cytoskeletal Fraction (4) } & 2 & 0.0 & 0.0 & 79.2 & 0.0 & 0.0 & 0.0 \\
\hline & 24 & 0.5 & 0.0 & 100.0 & 2.8 & 25.7 & 16.5 \\
\hline
\end{tabular}

Values are percentages (\%) of total cellular accumulation after 2 and $24 \mathrm{hr}$ of analyses performed in duplicate

Table 2. Time dependence of total cellular accumulation of 6 TKIs

\begin{tabular}{lcc}
\hline \multirow{2}{*}{ Drug } & \multicolumn{2}{c}{ Total cellular accumulation (fmol/mg protein) } \\
\cline { 2 - 3 } & 2 hours & 24 hours \\
\hline Sunitinib & 25692.8 & 4218.4 \\
Crizotinib & 5581.4 & 19867.6 \\
Erlotinib & 314.9 & 88.4 \\
Gefitinib & 1572.8 & 462.9 \\
Sorafenib & 2056.2 & 267.1 \\
Dasatinib & 244.4 & 97.1 \\
\hline
\end{tabular}

Values are means of analyses performed in duplicate. SEM was below $15 \%$

Crizotinib showed a similar distribution pattern compared to sunitinib. After 2 hours it was highly distributed into the cytosol (58.3\%), membranes and membrane organelles (41.7\%). Although the distribution remained consistent after 24 hours a 4.5 -fold increase in accumulation was observed.

One TKI, which showed a totally different distribution pattern, was erlotinib (Table 1). A distribution of erlotinib throughout the whole cell was observed after 2 hours exposure, although most of the accumulated erlotinib was located in cytoskeleton components. After 24 hours total accumulation decreased 20 -fold and was located solely in the cytoskeleton.

Gefitinib, which also inhibits EGFR showed a completely different pattern both after 2 and 24 hours exposure (Table 1). After 2 hours gefitinib was located in the cytosol (38.8\%), membranes and membrane 
organelles (44.4\%) and nuclear proteins (16.8\%), but not in the cytoskeleton as erlotinib, which hardly changed after 24 hours.
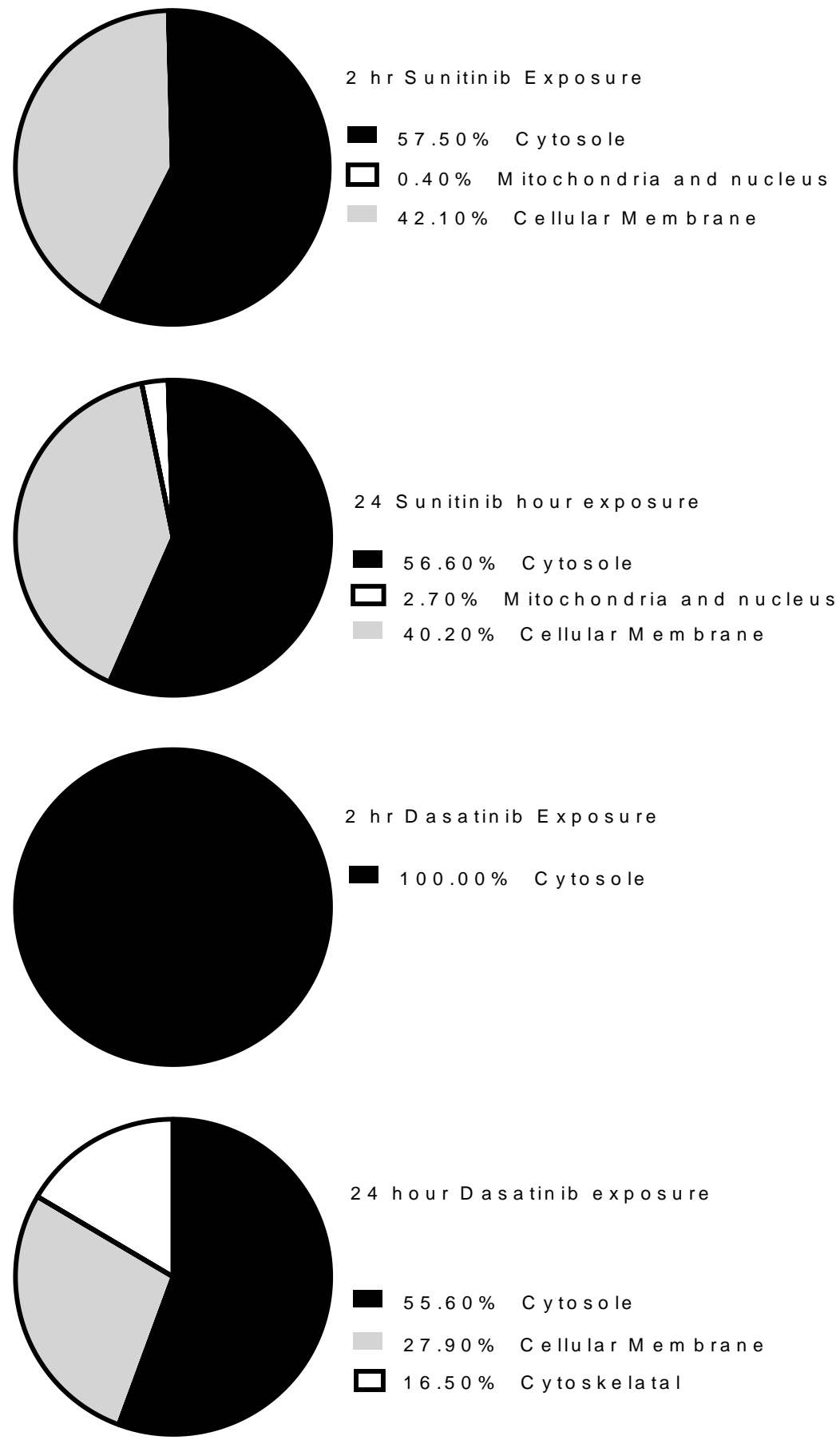

Figure 1. Pie chart of the cellular distribution of sunitinib and dasatinib after 2 and 24 hours exposure to 10 $\mu \mathrm{M}$ of either sunitinib or dasatinib respectively

Both sorafenib and dasatinib do not correspond with the other TKIs and each other's distribution pattern (Table 1; Figure 1). After two hours exposure, sorafenib is solely distributed in the membranes, membrane organelles (84.7\%) and cytosol (15.3\%). After 24 hours accumulation decreased 9.9-fold (Table 2) and sorafenib distribution shifted to membranes and membrane organelles (60.4\%), cytoskeleton (25.7\%) and nuclear proteins (13.9\%).

Accumulated dasatinib was located solely in the cytosol after two hours exposure (Figure 1; Table 1). 
Similar to sorafenib, the distribution of dasatinib changed completely after 24 hours. Some of the accumulated dasatinib shifted from the cytosol to membranes and membrane organelles (27.9\%) and cytoskeleton (16.5\%). There was only a small change in total accumulated drug.

Interestingly the total accumulation of the drugs was quite different both in the amount and the timecourse (Table 2). The absolute amount of sunitinib accumulated in the cells decreased by 3.9-fold from 2 to 24 hours which is in agreement with the decline of sunitinib accumulation in the temperature dependent accumulation study after 24 hours [14]. In contrast to sunitinib, crizotinib demonstrated a 4.5 -fold increase after 24 hours. Accumulation of erlotinib was very low and even decreased after 24 hours to $5.3 \%$ of that after 2 hours. Also, total sorafenib accumulation decreased considerably from 2 to 24 hours. In contrast to the five other TKIs, the total absolute amount of dasatinib accumulation in the cell hardly changed after 24 hours compared to 2 hours.

\section{Discussion}

The data show a large difference in both the cellular uptake and distribution. Structurally the TKIs investigated differ significantly (Figure 2) with a SMILE correlations of less than 0.4 with a median of 0.206538 (range $0.154453-0.389155$ ) as calculated by ChemMine, Table 3 [15]. No relationship could be determined between the cellular localization of the molecules to their structural makeup. The nature of the accumulation of these compounds is highly dependent on the degree to which they are lysosomally accumulated, our data demonstrates that sunitinib and crizotinib are in agreement with the physical properties of lysosomal accumulation. For sunitinib, the lysosomal accumulation has also been demonstrated by using its fluorescent properties which co-localized with the Lysotracker, a marker for lysosomes [7]. Moreover, pre-incubation with Bafilomycin-A prevented trapping of sunitinib in the lysosomes $[7,16]$. Sunitinib achieves very high cellular concentrations since it has been shown that $90 \%$ of accumulated drug is held within lysosomes within the cell structure. The cell survives this accumulation since the bulk of the drug is isolated away from its targets. The relationship between physiochemical properties and the observed absorption characteristics is currently under investigation and out of the scope of this paper.

Table 3. Comparison of molecular structures for crizotinib, dasatinib, erlotinib, gefitinib, sorafenib and sunitinib using ChemMine correlation of SMILEs.

\begin{tabular}{|l|l|l|l|l|l|l|}
\hline & Crizotinib & Dasatinib & Erlotinib & Gefitinib & Sorafenib & Sunitinib \\
\hline Crizotinib & & 0.231458 & 0.196302 & 0.285714 & 0.156522 & 0.203147 \\
\hline Dasatinib & 0.231458 & & 0.208279 & 0.296345 & 0.154453 & 0.275956 \\
\hline Erlotinib & 0.196302 & 0.208279 & & 0.389155 & 0.17601 & 0.206538 \\
\hline Gefitinib & 0.285714 & 0.296345 & 0.389155 & & 0.18059 & 0.262319 \\
\hline Sorafenib & 0.156522 & 0.154453 & 0.17601 & 0.18059 & & 0.191546 \\
\hline Sunitinib & 0.203147 & 0.275956 & 0.206538 & 0.262319 & 0.191546 & \\
\hline
\end{tabular}


<smiles>Cc1nc(Nc2ncc(C(=O)Nc3c(C)cccc3Cl)s2)cc(N2CCN(CCO)CC2)n1</smiles>

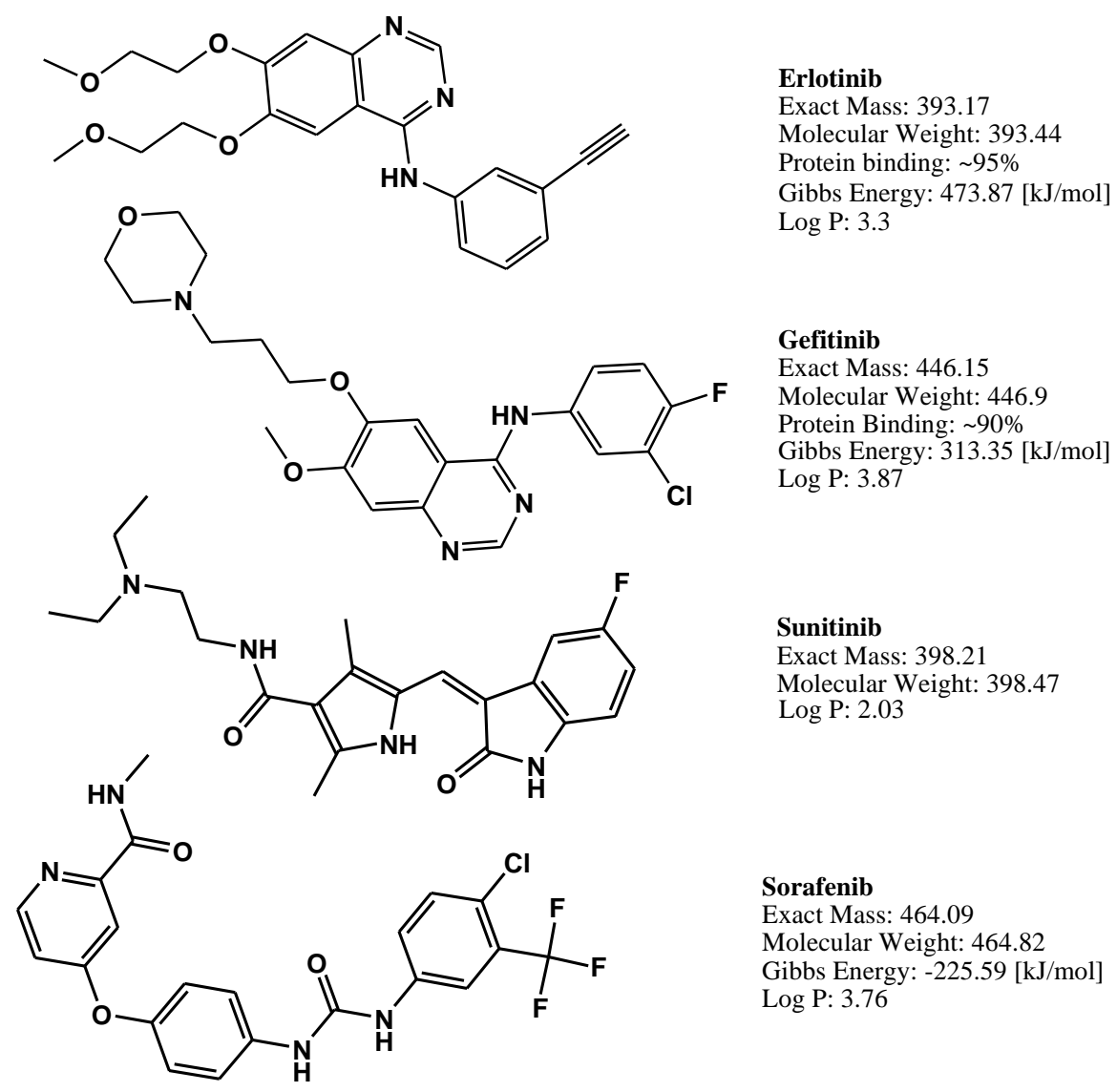

Figure 2. The molecular structures and know physical chemical properties for the six tyrosine kinase inhibitors under investigation

Also, for crizotinib we demonstrated that Bafilomycin prevented uptake into lysosomes (unpublished data). Trapping of these drugs is possibly mediated by a selective uptake of the drugs via multidrug resistance proteins, subsequently the drugs are protonated and cannot subsequently be effluxed anymore $[17,18]$. For erlotinib it is likely that it is transported into the membrane, binds to its target, but is effluxed before it can enter the cell [19]. In contrast, the other EGFR targeted drug (gefitinib) is taken up by an active transporter $[13,20,21]$ and subsequently trapped in the cell. Sorafenib is not only actively being transported into the cell but is possibly also trapped in the cell, either as the parent drug or as a metabolite, since it is substrate for various Phase I and Phase II enzymes [22,23]. It seems to be effluxed efficiently from the cell. Lastly, dasatinib is taken up by the cell, but is not further distributed and is not effluxed efficiently either. However, its cytosolic localization is in line with its high efficacy since its target BCR-abl (only in chronic myeloid leukemia) is located in the cytosol [1]. 


\section{Conclusion}

Protein fractions contained considerable TKI levels that changed markedly over time. This could be demonstrated by using the ProteoExtractR Subcellular Proteome Extraction kit. In addition, it can be concluded that the distribution of the six TKIs differs a lot and also changed over time. This may be related to the different physio-chemical properties of the various TKIs, favouring either a lysosomal accumulation or a rapid efflux from the cell [10]. Nevertheless, sunitinib and crizotinib distribution show comparable patterns, probably due to the lysosomal accumulation.

\section{References}

[1] F. Broekman, E. Giovannetti, G.J. Peters. Tyrosine kinase inhibitors: Multi-targeted or singletargeted? World J. Clin. Oncol. 2 (2011) 80-93.

[2] R. Chebib, L. Verlingue, N. Cozic, M. Faron, P. Burtin, V. Boige, A. Hollebecque, D. Malka. Angiogenesis inhibition in the second-line treatment of metastatic colorectal cancer: A systematic review and pooled analysis. Seminars in Oncology 44 (2017) 114-128.

[3] P. Wu, T.E. Nielsen, M.H. Clausen. FDA-approved small-molecule kinase inhibitors. Trends in Pharmacological Sciences 36 (2015) 422-439.

[4] M.P. Pinto, G. I. Owen, I. Retamal, M. Garrido. Angiogenesis inhibitors in early development for gastric cancer. Expert Opinion on Investigational Drugs 26 (2017) 1007-1017.

[5] C.G. Da Silva, R. J. Honeywell, H. Dekker, G. J. Peters. Physicochemical properties of novel protein kinase inhibitors in relation to their substrate specificity for drug transporters. Expert Opin. Drug Metab. Toxicol. 11 (2015) 703-717.

[6] N. Van Der Steen, C. Caparello, C. Rolfo, P. Pauwels, G.J. Peters, E. Giovannetti. New developments in the management of non-small-cell lung cancer, focus on rociletinib: What went wrong? OncoTargets and Therapy 9 (2016) 6065-6074.

[7] K.J. Gotink, H.J. Broxterman, M. Labots, R.R. De Haas, H. Dekker, R.J. Honeywell, M.A. Rudek, L.V. Beerepoot, R.J. Musetrs, G. Jansen, A.W. Griffioen,Y.G. Assaraf, R. Pili, G.J. Peters, H.M.W. Verheul. Lysosomal sequestration of sunitinib: A novel mechanism of drug resistance. Clin. Cancer Res. 17 (2011) 7337-7346.

[8] E. Giovannetti, M. Labots, H. Dekker, E. Galvani, J.S.W. Lind, R. Sciarillo, R. Honeywell, E.F. Smit, H.M.W. Verheul, G.J. Peters. Molecular Mechanisms and Modulation of Key Pathways Underlying the Synergistic Interaction of Sorafenib with Erlotinib in Non-Small-Cell-Lung Cancer (NSCLC) Cells | BenthamScience. Curr. Pharm. Des. (2013) 927-939.

[9] N.P. van Erp, H. Gelderblom, H.-J. Guchelaar. Clinical pharmacokinetics of tyrosine kinase inhibitors. Cancer Treat Rev 35 (2009) 692-706.

[10] K. Valko. Bio-mimetic chromatography to predict drug distribution in vivo. European Pharmaceutical Review (2010).

[11] A.D. Adema, K. Smid, N. Losekoot, R. Honeywell, H.M.W. Verheul, F. Mihren, M.L. Sandvold, G.J. Peters. Metabolism and accumulation of the lipophilic deoxynucleoside analogs elacytarabine and CP-4126. Invest. New Drugs 30 (2012) 1908-1916.

[12] R.J. Honeywell, D. Sarkisjan, I. Kathmann, M.H. Kristensen, G.J. Peters. Sensitive liquid chromatography mass spectrometry (LC-MS) assay reveals novel insights on DNA methylation and incorporation of gemcitabine, its metabolite difluorodeoxyuridine, deoxyuridine, and RX-3117 into DNA. Nucleosides, Nucleotides and Nucleic Acids 35 (2016) 652-662.

[13] R. Honeywell, C. Fatmawati, M. Buddha, S. Hitzerd, I. Kathman, G.J. Peters. Adaptation of a human gut epithelial model in relation to the assessment of clinical pharmacokinetic parameters for selected tyrosine kinase inhibitors. ADMET DMPK 3 (2015) 51-67.

[14] T. Rabilloud, C. Adessi, A. Giraudel, J. Lunardi. Improvement of the solubilization of proteins in twodimensional electrophoresis with immobilized pH gradients. Electrophoresis 18 (1997) 307-316. 
[15] T.W.H. Backman, Y. Cao, T. Girke. ChemMine tools: an online service for analyzing and clustering small molecules. Nucleic Acids Res. 39 (2011) W486-W491.

[16] K. Azijli, K.J. Gotink, H.M. Verheul. The Potential Role of Lysosomal Sequestration in Sunitinib Resistance of Renal Cell Cancer. J. Kidney Cancer VHL 2 (2016) 195-203.

[17] B. Zhitomirsky, Y.G. Assaraf. Lysosomes as mediators of drug resistance in cancer. Drug Resist. Updat. 24 (2016) 23-33.

[18] B. Zhitomirsky, Y.G. Assaraf. Lysosomal sequestration of hydrophobic weak base chemotherapeutics triggers lysosomal biogenesis and lysosome-dependent cancer multidrug resistance. Oncotarget 6 (2015) 1143-1156.

[19] R.J. Honeywell, S. Hitzerd, I. Kathmann, G. J. Peters. Transport of six tyrosine kinase inhibitors: active or passive? ADMET DMPK 4 (2016) 23-34.

[20] C. Neul, E. Schaeffeler, A. Sparreboom, S. Laufer, M. Schwab, A.T. Nies. Impact of Membrane Drug Transporters on Resistance to Small-Molecule Tyrosine Kinase Inhibitors. Trends in Pharmacological Sciences 37 (2016) 904-932.

[21] M. Galetti, P. Petronini, C. Fumarola, D. Cretella, S. La Monica, M. Bonelli, A. Cavazzoni, F. Saccani, C. Caffarra, R. Andreoli, A. Mutti, M. Tiseo, A. Ardizzoni, R.R. Alfieri. Effect of ABCG2/BCRP Expression on Efflux and Uptake of Gefitinib in NSCLC Cell Lines. PLoS One 10 (2015) e0141795.

[22] E.I. Zimmerman, S. Hu, J.L. Roberts, A.A. Gibbson, S.J. Orwick, L. Li, A. Sparreboom, S.D. Baker. Contribution of OATP1B1 and OATP1B3 to the disposition of sorafenib and sorafenib-glucuronide. Clin. Cancer Res. 19 (2013) 1458-66.

[23] S.D. Baker, S. Hu. Pharmacokinetic considerations for new targeted therapies. Clin. Pharmacol. Ther. 85 (2009) 208-11. 